\title{
Variabilidade espacial da fertilidade do solo em área cultivada com algodoeiro no Cerrado do Brasil
}

\author{
João H. Zonta ${ }^{1}$, Ziany N. Brandão ${ }^{2}$, José da C. Medeiros ${ }^{3}$, Ronei S. Sana ${ }^{4} \&$ Valdinei Sofiatti $^{5}$
}

${ }^{1}$ Embrapa Algodão. Campina Grande, PB. Fone: (83) 3182-4412. E-mail: joao-henrique.zonta@embrapa.br (Autor correspondente)

${ }^{2}$ Embrapa Algodão. Campina Grande, PB. Fone: (83) 3182-4412. E-mail: ziany.brandao@embrapa.br

${ }^{3}$ Embrapa Algodão. Campina Grande, PB. Fone: (83) 3182-4412. E-mail: jose.medeiros@embrapa.br

${ }^{4}$ SLC Agrícola. Porto Alegre, RS. Fone: (51) 3230-7870. E-mail: ronei.sana@slcagricola.com.br

${ }^{5}$ Embrapa Algodão. Campina Grande, PB. Fone: (83) 3182-4412. E-mail: valdinei.sofiatti@embrapa.br

\section{Palavras-chave:}

krigagem

geoestatística

aplicação taxa variável

\section{R E S U M O}

Propôs-se, neste trabalho, avaliar a variabilidade espacial dos atributos de fertilidade do solo em Latossolo Vermelho Amarelo em plantio direto para o algodoeiro em Cristalina, GO, visando à adoção da agricultura de precisão no manejo da área. A grade amostral foi constituída de 90 pontos $(80 \times 80 \mathrm{~m})$ distribuídos numa área de 57,6 ha. Foram determinados os teores de $\mathrm{P}, \mathrm{K}, \mathrm{Ca}, \mathrm{Mg}, \mathrm{S}, \mathrm{Na}, \mathrm{Cu}, \mathrm{Mn}, \mathrm{B}, \mathrm{Zn}, \mathrm{Fe}$, matéria orgânica (MO), pH, soma de bases $(\mathrm{SB})$, saturação por bases $(\mathrm{V})$, saturação por alumínio $(\mathrm{m})$ e condutividade elétrica aparente do solo (CE) a 30 e $90 \mathrm{~cm}$ de profundidade. Efetuou-se a análise descritiva clássica e em seguida foram modelados semivariogramas para todos os atributos e mapas de krigagens. A dependência espacial das propriedades do solo analisadas indica que as variações espaciais devem ser consideradas no planejamento de coleta de amostras de solo e nas práticas de manejo do solo. Para a adoção da agricultura de precisão e aplicação de insumos em taxa variável, deve-se realizar um estudo criterioso da variabilidade da fertilidade do solo, gerando mapas por classe de fertilidade de cada atributo, o que permite identificar para quais atributos esta prática de manejo deve ser adotada.

\section{Key words:}

kriging

geostatistics

variable rate application

\section{Spatial variability of soil fertility in cultivated area of cotton in Brazilian Savannah}

\begin{abstract}
A B S T R A C T
The objective of this work was to analyze the spatial variability of fertility attributes in an Oxisol under tillage to cotton cultivation in Cristalina, GO, aiming the adoption of precision agriculture in the area management. The soil was collected using a sample grid, which consisted of 90 points $(80 \mathrm{x} 80 \mathrm{~m})$, distributed into 57.6 ha. Were determined contents of $\mathrm{P}, \mathrm{K}$, $\mathrm{Ca}, \mathrm{Mg}, \mathrm{S}, \mathrm{Na}, \mathrm{Cu}, \mathrm{Mn}, \mathrm{B}, \mathrm{Zn}, \mathrm{Fe}$, organic matter $(\mathrm{OM}), \mathrm{pH}$, sum of bases (SB), base saturation (V), aluminum saturation (m), as well as the apparent electrical conductivity of soil (EC) measured at 30 and $90 \mathrm{~cm}$ depth. Classic descriptive analysis was conducted and then, semivariograms were modeled for all attributes and krigging maps. The spatial dependence of analyzed soil properties indicates that the spatial variability should be considered during the planning of soil sampling and also to soil management practices. Concerning to precision agriculture adoption and the application of inputs in variable rates, it is required to do a careful study of the soil fertility variability, creating maps based on fertility classes for each attribute, allowing to identify for which attributes this management practice should be adopted.
\end{abstract}

\section{INTRODUÇÃO}

O cultivo do algodoeiro no Cerrado brasileiro tem obtido sucesso devido a diversos fatores, sendo os principais as condições climáticas favoráveis, topografia que permite a mecanização, os programas federais e estaduais de incentivo à cotonicultura e, especialmente, a intensiva adoção de tecnologias modernas. Devido à soma desses fatores, o cerrado brasileiro apresenta as maiores produtividades de algodão no Brasil e no mundo, em condições não irrigadas.

Em geral, as fazendas produtoras de algodão no cerrado brasileiro são gerenciadas por empresas agrícolas com sistema de produção intensivo, totalmente mecanizado e, em contrapartida, com produtividades elevadas; este tipo de sistema de produção gera um custo elevado especialmente com a adubação, controle de plantas daninhas e de pragas e doenças. A aplicação desses insumos (fertilizantes, herbicidas, inseticidas e fungicidas) é feita em grandes áreas de forma homogênea sem se considerar a variabilidade espacial das características do solo e da incidência de pragas e doenças. Esse tipo de manejo não é adotado somente no Brasil, mas também pela maioria dos produtores de algodão no mundo. Stewart et al. (2005) observaram que nos Estados Unidos os produtores de algodão fazem o manejo considerando grandes áreas como sendo homogêneas e desconsiderando a existência da variabilidade espacial. 
Com relação aos atributos do solo, diversos trabalhos realizados anteriormente têm demonstrado que a variabilidade espacial dessas características em áreas de exploração agrícola é de suma importância para a adoção de técnicas de agricultura de precisão (Serrano et al., 2010). Segundo Amado et al. (2009) o conhecimento da variabilidade espacial dos atributos do solo e da cultura é uma ferramenta significativa para analisar a variabilidade da produtividade e aperfeiçoar o manejo em áreas agrícolas, por meio de um gerenciamento agrícola que leve em consideração informações pontuais de solo e das culturas.

Normalmente, ao se analisar, na estatística clássica, os atributos do solo, a influência da heterogeneidade espacial sobre a representatividade dos valores médios é ignorada (Chaves \& Farias, 2009). Para resolver tal impasse torna-se imprescindível o uso de técnicas de geoestatística que, além de analisar a dependência espacial desses valores, ainda proporciona meios para que sejam efetuados os respectivos mapeamentos da área estudada, por meio da krigagem, uma vez que o modelo da dependência espacial seja conhecido por meio do semivariograma (Lemos Filho et al., 2008). O semivariograma é um gráfico que caracteriza a estrutura da variância do atributo estudado como variável regionalizada em função da distância no terreno.

Aliada a isto, a evolução da tecnologia na agricultura tem demonstrado a importância de se mensurar a variabilidade espacial e temporal das propriedades do solo que afetam a produtividade das culturas (Andrade et al., 2005; Vieira et al., 2007) visto que tais informações podem ser utilizadas como base para a aplicação de insumos em taxa variável e ajustada especificamente para cada parcela (Faria et al., 2009). Isso é possível somente através do estabelecendo de zonas específicas de manejo, condições necessárias à agricultura de precisão (Dalchiavon et al., 2012).

Segundo Werner et al. (2007) a adoção da agricultura de precisão (AP) se encontra na fase em que os agricultores buscam solucionar os principais problemas levantados em sua lavoura, através de mapas de produtividade e de fertilidade. Para tal, a AP concilia o uso de tecnologias para o manejo diferenciado do solo, dos insumos e da cultura, visando maximizar a produtividade.

A AP foi executada primeiramente no manejo de nutrientes, fundamentada em um esquema de coleta de solo em malha sendo, em geral, adotado um esquema de amostragem a cada $50 \mathrm{~m}$, ou seja, 4 amostras por hectare (Inamassu et al., 2011).

Neste contexto, a análise detalhada da variabilidade dos atributos do solo pode indicar alternativas de manejo para a redução dos efeitos da sua variabilidade sobre a produção das culturas; assim, devido tanto ao recém-surgimento como à inquestionável importância prática da $\mathrm{AP}$, ultimamente no Brasil os estudos geoestatísticos visando conhecer melhor a variabilidade dos atributos químicos no solo vêm ganhando cada vez mais, destaque e importância.

Desta forma, a geoestatística contribui para a AP com parâmetros de análise como a "dependência espacial" dos dados para fornecer um pouco mais de segurança no número de dados necessário e no tamanho da malha de coleta de amostras.
Neste contexto, o objetivo do trabalho foi analisar a variabilidade espacial dos atributos químicos de um Latossolo Vermelho Amarelo cultivado com algodão em uma fazenda comercial na região do Cerrado Brasileiro, visando à adoção da agricultura de precisão nas práticas de manejo da fertilidade do solo.

\section{Material e Métodos}

O experimento foi conduzido em área de cultivo de algodoeiro localizada no município de Cristalina, GO, com dimensão de 57,6 ha cujas coordenadas centrais são $16^{\circ} 10^{\prime} 16^{\prime \prime}$ S, $47^{\circ} 37^{\prime} 47^{\prime \prime}$. Os solos da região predominantes são Latossolos vermelho-amarelos sendo a altitude média na área experimental de $1.009 \mathrm{~m}$ com relevo plano e declividade média de $0,5 \%$. O clima da região é caracterizado como tropical com estação seca e predominância do tipo Aw, da classificação climática de Köppen, com temperaturas médias de 28,6 e $16,8^{\circ} \mathrm{C}$ para a máxima e mínima, respectivamente. O período chuvoso é de outubro a março com precipitação média anual de $1.446,5 \mathrm{~mm}$.

A área estava sendo manejada em sistema plantio direto/ cultivo mínimo desde a safra 2001/2002, com sucessão das culturas de soja/milho e algodão. Antes da instalação do experimento foi preparada uma grade amostral com 90 pontos no espaçamento de $80 \times 80 \mathrm{~m}$. Os pontos centrais foram marcados previamente e georreferenciados com um GPS (modelo 76 CSx marca Garmim). A coleta das amostras de solo foi realizada em novembro de 2010 após a colheita da soja antecedendo o plantio do algodoeiro correspondente à safra 2010/2011.

As medidas da condutividade elétrica aparente (CEa) foram realizadas para cada ponto amostral antecedendo a coleta das amostras do solo. A malha com 90 pontos (cada um espaçado $80 \mathrm{~m}$ ) correspondente a 0,64 ha serviu para a coleta de solos com vista à análise química tendo sido coletadas 8 subamostras no raio de até $10 \mathrm{~m}$ de cada ponto central. Este conjunto de amostras foi enviado ao Instituto Brasileiro de Análise (IBRA) para determinação dos atributos químicos do solo, conforme segue: a) macronutrientes: fósforo $(\mathrm{P})$, potássio $(\mathrm{K})$, cálcio $(\mathrm{Ca})$, magnésio $(\mathrm{Mg})$ e enxofre $(\mathrm{S}), \mathrm{b})$ micronutrientes: sódio $(\mathrm{Na})$, cobre $(\mathrm{Cu})$, manganês $(\mathrm{Mn})$, boro $(\mathrm{B})$, zinco $(\mathrm{Zn})$ e ferro $(\mathrm{Fe}), \mathrm{c})$ matéria orgânica $(\mathrm{MO}), \mathrm{pH}$, soma de bases $(\mathrm{SB})$. Foram calculadas a saturação por bases $(\mathrm{V})$ e a saturação por alumínio (m).

A condutividade elétrica aparente do solo (CEa) foi determinada a campo através do equipamento VERIS EC 3100 (Veris Technologies, 2011), adaptado a um sistema subsolador com eletrodos de contato, conforme proposto por Inamassu et al. (2007), com as medidas coletadas e armazenadas e georreferenciadas por um GPS Trimble. Os dados obtidos foram de latitude, longitude e altitude ( $\mathrm{m}$ ), além dos valores de CEa do solo $\left(\mathrm{mS} \mathrm{m}^{-1}\right)$, para as profundidades de 0-30 e 0-90 cm coletados a intervalos de um segundo.

Realizou-se a análise descritiva dos dados sendo calculadas as medidas de posição (média, mediana, máximo e mínimo), de dispersão (desvio padrão) e de forma da distribuição 
(coeficiente de variação, coeficiente de assimetria e coeficiente de curtose). A hipótese de normalidade dos dados foi verificada com o teste de Shapiro \& Wilk (1965) a nível de $5 \%$ de probabilidade. O coeficiente de variação (CV) foi considerado baixo quando $\mathrm{CV}<10 \%$ (homocedasticidade); médio, quando $10 \%<\mathrm{CV}<20 \%$; alto, quando $20 \%<\mathrm{CV}<$ $30 \%$ e muito alto quando $\mathrm{CV}>30 \%$ (heterocedasticidade) conforme Gomes \& Garcia (2002).

Após a verificação de prováveis tendências espaciais nos dados para estimar sua dependência espacial e identificar variações sistemáticas e randômicas, as semivariâncias experimentais foram então estimadas utilizando-se o software Vesper 1.6 (Minasny et al., 2006). Os modelos de ajuste do semivariograma foram selecionados com base no menor valor da raiz quadrada do erro médio (RQEM) e de AIC (Critério de Informação de Akaike (Akaike, 1973).

O grau de dependência espacial dos semivariogramas foi avaliado pelo grau de dependência espacial (GD) segundo os intervalos propostos por Cambardella et al. (1994): GD < 25\% - forte dependência espacial; 25\% < GD < 75\% - moderada dependência espacial e GD > 75\% - fraca dependência espacial. $\mathrm{O}$ valor de GD é dado pela Eq. 1:

$$
\mathrm{GD} \%=\frac{\mathrm{C}_{0}}{\mathrm{C}_{0}+\mathrm{C}_{1}}
$$

em que:

$$
\begin{array}{ll}
C_{0} & \text { - efeito pepita } \\
C_{1} & \text { - variância estrutural }
\end{array}
$$

Após o ajuste dos modelos que melhor descrevem os semivariogramas e a identificação da dependência espacial, foi utilizado o método de krigagem em blocos (10 x $10 \mathrm{~m})$ para estimar os valores nos pontos não medidos na área e gerar os mapas temáticos com uso do software Vesper 1.6 (Minasny et al., 2006) e Quantun Gis 1.7.4 (Quantum Gis Development Team, 2012).

\section{RESUltados e Discussão}

$\mathrm{Na}$ Tabela 1 são apresentados os dados da análise exploratória para as características do solo analisadas. As medidas de tendência central (média e mediana) foram similares em pelo menos $75 \%$ das características avaliadas indicando a distribuição dos dados com pequena variação em torno do valor central.

Segundo Dalchiavon et al. (2012) a variabilidade de um atributo pode ser classificada de acordo com a magnitude de seu coeficiente de variação $(\mathrm{CV})$ classificando-o como baixo (CV < 10\%), médio $(10 \%<\mathrm{CV}<20 \%)$, alto $(20 \%<\mathrm{CV}<30 \%)$ e muito alto $(\mathrm{CV}>30 \%)$. Observa-se, com base nos resultados apresentados na Tabela 1, que para as características de solo avaliadas, exceto para o $\mathrm{P}, \mathrm{Ca}, \mathrm{Mg}$ e $\mathrm{Cu}$ (alto e muito alto), os coeficientes de variação $(\mathrm{CV})$ das demais características do solo foram classificados médio ou baixo.

Esta variação entre os CV's dos diferentes atributos do solo é esperada visto que, segundo Carvalho et al. (2003), a variabilidade dos atributos químicos do solo é consequência de complexas interações dos processos de sua formação e de práticas de manejo do solo e da cultura com impacto sobretudo nas camadas superficiais do solo. Este comportamento das propriedades do solo na área pode, também, ser explicado pelas sucessivas e irregulares adubações e calagens que vêm sendo aplicadas na área, ao longo dos cultivos (Silva \& Chaves, 2001).

A variabilidade para o fósforo foi classificada muito alta, provavelmente pelo fato de a adubação fosfatada ser realizada na linha de plantio o que culmina na sua desuniformidade na área, como um todo, em razão da baixa mobilidade do fósforo no solo. Motomiya et al. (2011) também observaram, estudando a variabilidade espacial dos atributos do solo em área de cultivo

\begin{tabular}{|c|c|c|c|c|c|c|c|c|c|}
\hline \multirow{2}{*}{ Atributo $^{1}$} & \multicolumn{9}{|c|}{ Estatística descritiva } \\
\hline & Média & Mediana & Máximo & Mínimo & $\mathrm{DP}^{2}$ & $\mathrm{CV}^{3}{ }^{3}$ & $\mathrm{C}_{\mathrm{k}}^{4}$ & $\mathrm{C}_{\mathrm{A}}{ }^{5}$ & $w^{6}$ \\
\hline$P\left(\mathrm{mg} \mathrm{dm}^{-3}\right)$ & 2,62 & 2,60 & 4,70 & 0,60 & 1,03 & 39,49 & $-0,80$ & 0,22 & ns \\
\hline $\mathrm{K}\left(\mathrm{cmol}_{\mathrm{c}} \mathrm{dm}^{-3}\right)$ & 0,23 & 0,24 & 0,30 & 0,18 & 0,02 & 12,01 & $-0,50$ & 0,17 & ns \\
\hline $\mathrm{Ca}\left(\mathrm{cmol}_{\mathrm{c}} \mathrm{dm}^{-3}\right)$ & 3,06 & 2,90 & 4,80 & 1,80 & 0,64 & 21,18 & $-0,18$ & 0,45 & * \\
\hline $\mathrm{Mg}\left(\mathrm{cmol}_{\mathrm{c}} \mathrm{dm}^{-3}\right)$ & 1,08 & 1,10 & 1,80 & 0,60 & 0,26 & 24,31 & $-0,58$ & 0,27 & * \\
\hline Cu (mg dm-3) & 1,50 & 1,40 & 2,70 & 0,70 & 0,48 & 31,91 & $-0,13$ & 0,67 & * \\
\hline $\mathrm{Mn}\left(\mathrm{mg} \mathrm{dm}^{-3}\right)$ & 2,07 & 2,00 & 3,00 & 1,60 & 0,40 & 19,24 & $-0,08$ & 0,85 & * \\
\hline $\mathrm{B}\left(\mathrm{mg} \mathrm{dm}^{-3}\right)$ & 1,21 & 1,21 & 1,66 & 0,72 & 0,23 & 18,88 & $-0,64$ & 0,04 & ns \\
\hline $\mathrm{Zn}\left(\mathrm{mg} \mathrm{dm}^{-3}\right)$ & 3,91 & 3,90 & 5,00 & 3,00 & 0,50 & 12,93 & $-0,67$ & 0,25 & * \\
\hline $\mathrm{MO}\left(\mathrm{g} \mathrm{dm}^{-3}\right)^{\prime}$ & 2,90 & 2,90 & 3,50 & 2,30 & 0,26 & 9,10 & $-0,50$ & $-0,01$ & ns \\
\hline $\mathrm{H}+\mathrm{Al}\left(\mathrm{cmol}_{\mathrm{c}} \mathrm{dm}^{-3}\right)$ & 2,58 & 2,60 & 3,60 & 0,80 & 0,50 & 19,38 & 0,87 & $-0,58$ & * \\
\hline $\mathrm{pH}$ & 5,33 & 5,40 & 6,20 & 4,60 & 0,34 & 6,47 & 0,12 & 0,05 & * \\
\hline $\mathrm{SB}\left(\mathrm{cmol}_{\mathrm{C}} \mathrm{dm}^{-3}\right)$ & 4,26 & 4,25 & 5,92 & 2,81 & 0,77 & 18,29 & $-0,67$ & 0,08 & ns \\
\hline $\mathrm{m} \%$ & 0,22 & 0,22 & 0,29 & 0,16 & 0,03 & 13,88 & $-0,79$ & 0,08 & ns \\
\hline V\% & 62,7 & 62,81 & 91,6 & 44,78 & 8,63 & 13,77 & 0,43 & 0,28 & ns \\
\hline CE rasa $\left(\mathrm{mS} \mathrm{m}^{-1}\right)$ & 10,06 & 9,92 & 13,82 & 7,61 & 1,04 & 10,42 & 1,50 & 0,79 & * \\
\hline CE prof $\left(\mathrm{mS} \mathrm{m}^{-1}\right)$ & 5,46 & 5,40 & 6,45 & 4,81 & 0,42 & 7,71 & $-0,41$ & 0,49 & * \\
\hline
\end{tabular}

Tabela 1. Parâmetros da estatística descritiva de alguns atributos químicos do solo em área cultivada com algodoeiro (90 amostras)

1 - P, K, Ca, Mg, Cu, Mn, B, Zn, MO, H+Al, SB, m\%, V\%, CE rasa e Ceprof são, respectivamente, o fósforo, potássio, cálcio, magnésio, cobre, manganês, boro, zinco, matéria orgânica, hidrogênio + alumínio, soma de bases, saturação por alumínio, saturação por bases, condutividade elétrica aparente a 30 e a $60 \mathrm{~cm}$. ${ }^{2} \mathrm{DP}-\mathrm{Desvio}$ padrão; ${ }^{3} \mathrm{CV}$ - Coeficiente de variação, ${ }^{4} \mathrm{CK}-\mathrm{Coeficiente}$ de curtose; ${ }^{5} \mathrm{C}_{\mathrm{A}}$ - Coeficiente de assimetria; ${ }^{6} \mathrm{~W}$ - Teste de Shapiro-Wilk's; * - Distribuição não-normal pelo teste de Shapiro-Wilk's (p-valor < 0,05); ${ }^{\text {ns }}$ - Distribuição normal pelo teste de ShapiroWilk's ( $p$-valor $>0,05)$ 
de algodoeiro no Cerrado brasileiro, alto CV para o fósforo sendo o mesmo igual a $38,6 \%$, resultado condizente com os aqui apresentados.

Ao analisar a dinâmica de alguns atributos do solo nota-se, de acordo com Carvalho et al. (2011) que para o cultivo do algodoeiro o solo apresenta adequado a alto teor de K, baixo teor de $\mathrm{P}$ e pH ácido, saturação por bases e alumínio adequada (solos eutróficos), teor médio de matéria orgânica em solos de Cerrado (1,5 a 3\%) e valores considerados altos dos micronutrientes B, Zn, Mn e Cu; constatou-se, ainda, seguindo a classificação proposta pela CFSG (1988) que o $\mathrm{Ca}\left(>2,5 \mathrm{cmol}_{\mathrm{c}} \mathrm{dm}^{-3}\right)$ e o $\mathrm{Mg}$ $\left(>0,6 \mathrm{cmol}_{\mathrm{c}} \mathrm{dm}^{-3}\right)$ estavam presentes em alta disponibilidade.

Com relação à CErasa e CEprof, que podem ser utilizadas como estimadores das condições da fertilidade do solo e sua variabilidade, os valores apresentados são considerados altos visto que Aimrun et al. (2007) encontraram valores de CE de 5,2 e $5,67 \mathrm{mS} \mathrm{m}^{-1}$, respectivamente; esses altos valores podem estar ligados às frequentes adubações que são realizadas na área de vez que a mesma vem sendo cultivada em sistema de alta tecnologia, há vários anos.

Com base nos resultados, observa-se que os valores de $\mathrm{P}$, $\mathrm{K}, \mathrm{MO}, \mathrm{B}, \mathrm{SB}, \mathrm{m} \%$ e V\% apresentaram distribuição normal de acordo com o teste de Kolmogorov-Smirnov. Os outros parâmetros apresentaram coeficientes de assimetria elevados e/ou curtose e, desta forma, apresentaram distribuição nãonormal pelo mesmo teste; com exceção das características MO e $\mathrm{H}+\mathrm{Al}$, as demais apresentaram assimetria positiva.

Chaves \& Farias (2009) ainda afirmam que, mesmo que os dados não apresentem distribuições simétricas e se os coeficientes de assimetria e curtose forem próximos de zero, tal como valores médios e medianos próximos, como é o caso da maioria dos atributos neste solo, tratamentos geoestatísticos podem ser aplicados possibilitando avaliar a dependência espacial dos atributos haja vista que na geoestatística mais importante que anormalidade dos dados é a ocorrência ou não do efeito proporcional em que a média e a variância dos dados não sejam constantes na área de estudo.
Na Tabela 2 se encontram os parâmetros dos semivariogramas ajustados podendo-se constatar que todos os atributos avaliados apresentaram dependência espacial. A dependência espacial denotou que o comportamento dessas variáveis regionalizadas não foi aleatório e que as distâncias entre pontos, utilizadas na malha geoestatística, foram suficientes para o estudo da variabilidade espacial nesta área.

Os modelos foram ajustados para os semivariogramas experimentais em função do seu melhor ajuste escolhido em função do menor valor da Raiz Quadrada do Erro Médio (RQEM) e do critério de Akaike (AIC). Com exceção dos atributos $\mathrm{Ca}$ e $\mathrm{CErasa}$ e $\mathrm{H}+\mathrm{Al}$ cujos semivariogramas foram ajustados seguindo os modelos Gaussiano e Exponencial, respectivamente, para os demais atributos, os semivariogramas experimentais foram ajustados seguindo o modelo Esférico (Tabela 2). Na Figura 1 são apresentados, como forma de ilustração, os semivariogramas ajustados para o fósforo (Esférico) e cálcio (Gaussiano).

Esses resultados são semelhantes aos obtidos por Dalchiavon et al. (2012) que, estudando a dependência espacial dos atributos da fertilidade de um latossolo distroférrico, obtiveram ajuste do semivariograma através do modelo esférico em $70 \%$ dos atributos avaliados, como $\mathrm{P}, \mathrm{Mg}, \mathrm{MO}, \mathrm{pH}$ e $\mathrm{m} \%$. Motomiya et al. (2011 também obtiveram, estudando a variabilidade espacial dos atributos de um solo cultivado com algodão no Cerrado do Brasil, para os atributos químicos do solo, os melhores ajustes para os semivariogramas através do modelo esférico.

Na Tabela 2 podem ser visualizados, também, os valores dos alcances geoestatísticos dos atributos que ficaram entre 264,5 m (B) e 84,28 m (CEprof) sendo que para a maioria dos atributos o alcance variou na faixa entre 100 e $200 \mathrm{~m}$, conforme $\mathrm{o}$ atributo analisado. Motomiya et al. (2011) encontraram, em solos do Cerrado, alcances de 118, 200, 50, 124 e 127 m para $\mathrm{SB}, \mathrm{K}, \mathrm{H}+\mathrm{Al}$, Ca e $\mathrm{pH}$, respectivamente, valores próximos ao encontrados no presente trabalho.

O alcance é o principal parâmetro fornecido pela geoestatística a qual representa a distância em que uma

Tabela 2. Modelos e parâmetros estimados dos semivariogramas de alguns atributos químicos do solo em área de algodoeiro

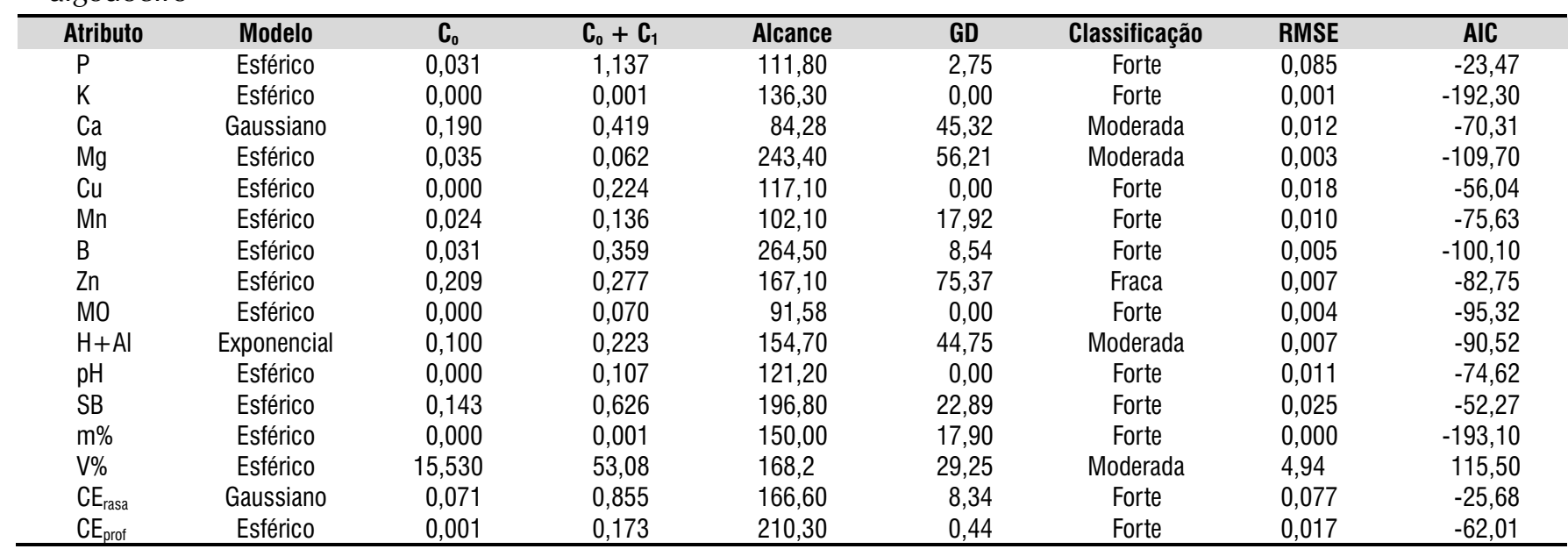

$C_{0}$. Efeito pepita; $C_{0}+C_{1}$. patamar; GD - Grau de dependência espacial $\left(C_{1} / C 0+C_{1}\right)$ 
A. Cálcio

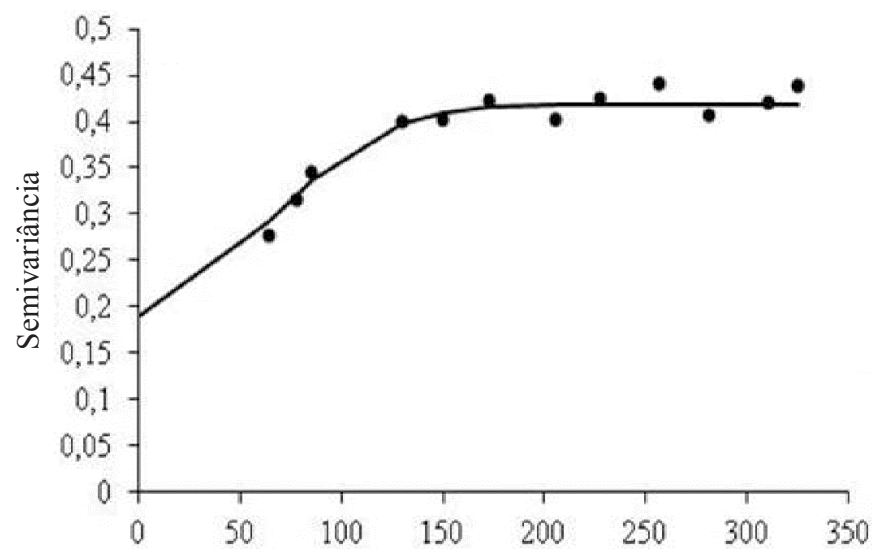

B. Fósforo

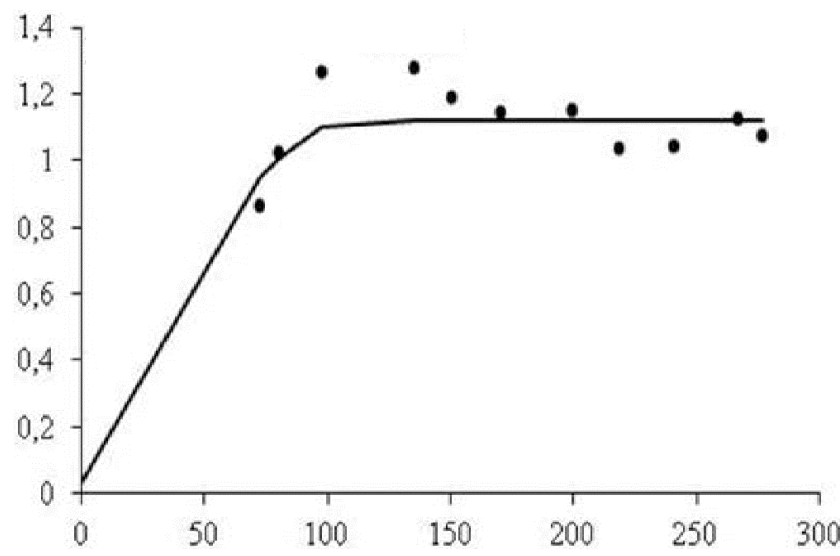

Distância (m)

Figura 1. Semivariogramas ajustados para os atributos cálcio (A) e fósforo (B)

variável sinaliza continuidade espacial sendo que, a partir desta distância, o comportamento espacial da variável passa a ser totalmente aleatório (Lemos Filho et al., 2008). Desta forma, sua determinação é de suma importância para a agricultura de precisão uma vez que os programas computacionais o utilizam para poder monitorar o maquinário agrícola quando da aplicação de insumos com taxas variáveis (Dalchiavon et al., 2011).

Ainda de acordo com Corá \& Beraldo (2006) muitos consultores de agricultura de precisão no Brasil não levam em consideração a dependência espacial dos atributos do solo para a coleta de dados e geração dos mapas temáticos sendo utilizados métodos baseados em interpolação linear ou polinomial, o que reduz a precisão dos mapas de manejo.

Para o solo em estudo, por exemplo, as amostras poderiam ser coletadas para aplicação em taxa variável de fósforo, com uma grade amostral de até $112 \times 112 \mathrm{~m}$ ou uma amostra para cada 1,25 ha enquanto que para a aplicação de calcário em taxa variável em função da soma de bases (SB), a grade amostral poderia ser de até $196 \times 196 \mathrm{~m}$ ou uma amostra a cada 3,85 ha. O interessante é que todos os valores de alcance obtidos foram maiores que o valor de espaçamento entre as amostragens (80 $\mathrm{m}$ ), indicando que as amostras estão correlacionadas umas às outras, permitindo que se façam interpolações (Vieira et al., 2007).

Quanto à relação a dependência espacial, $\mathrm{P}, \mathrm{K}, \mathrm{Cu}, \mathrm{Mn}$, $\mathrm{B}, \mathrm{MO}, \mathrm{pH}, \mathrm{SB}, \mathrm{m} \%$, CErasa e CEprof apresentaram forte dependência espacial, $\mathrm{Ca}, \mathrm{Mg}$, $\mathrm{V} \%$ e pH moderada dependência espacial e o Zn fraca dependência espacial. Silva \& Chaves (2001) também observaram forte dependência espacial para o fósforo e o potássio no solo. Motomiya et al. (2011) não encontraram dependência especial para $\mathrm{Pe} \mathrm{Mg}$ nem dependência moderada para outros atributos, como $\mathrm{K}, \mathrm{pH}, \mathrm{H}+\mathrm{Al}, \mathrm{SB}, \mathrm{V} \%$, entre outros, o que demonstra que o grau de dependência espacial dos atributos do solo pode variar em função da área e do manejo aos quais é submetida.

De acordo com Cambardella et al. (1994) as variáveis que apresentam forte dependência especial são mais influenciadas pelas propriedades intrínsecas do solo, como textura e mineralogia, enquanto aquelas que apresentam fraca dependência são influenciadas por propriedades extrínsecas do solo, como o cultivo e a aplicação de fertilizantes, ou seja, são dependentes do manejo do solo. Desta forma e de acordo com os resultados, pode-se observar que os valores de $\mathrm{Ca}, \mathrm{Mg}, \mathrm{V} \% \mathrm{e}$ $\mathrm{H}+\mathrm{Al}$ podem ter sido influenciados pela aplicação desuniforme de calcário na área visto que apresentam dependência espacial moderada (Tabela 2) e ambos estão relacionados à aplicação deste corretivo.

A partir do ajuste dos semivariogramas foram construídos mapas de krigagem, fundamentais para a agricultura de precisão haja vista que a partir desses são definidas zonas específicas de manejo da fertilidade do solo possibilitando a aplicação de insumos com taxas variáveis visando à homogeneização da fertilidade do solo.

Na Figura 2 são apresentados os mapas de krigagem para os atributos $\mathrm{K}$ e $\mathrm{P}$, Ca e $\mathrm{Mg}$ e $\mathrm{V} \%$ e $\mathrm{pH}$, sendo os mapas de krigagem elaborados de acordo com as classes de fertilidade do solo recomendadas por Carvalho et al. (2011). Segundo Zanão Júnior et al. (2010), este critério é mais coerente do que fazê-los conforme as classes sugeridas pelos programas que os geram, visto que as recomendações de adubação são realizadas de acordo com as classificações dos níveis dos nutrientes no solo.

De acordo com a Figura 2B, o solo apresentou muito baixo teor de $\mathrm{P}\left(\mathrm{P}<5,0 \mathrm{mg} \mathrm{dm}^{-3}\right)$ em $100 \%$ da área (Carvalho et al., 2011). Este nutriente assume grande importância na produção agrícola de vez que se trata de um dos nutrientes mais limitantes à produtividade das culturas. Desta forma, verifica-se a necessidade de se realizar a adubação fosfatada para que sejam corrigidas as deficiências de $\mathrm{P}$ no solo visando à obtenção de elevadas produtividades. Como não se constatou variabilidade quando categorizado em classes de fertilidade nos níveis de fósforo no solo, a aplicação deste nutriente em taxa variável não seria, neste caso, recomendada. Bronson et al. (2003) verificaram, analisando dois campos cultivados com algodão irrigado, que quando foi aplicado fertilizante fosfatado em taxa variável, taxa constante e sem aplicação de fertilizante não houve resposta significativa na produtividade apesar de uma redução no uso de fertilizantes. Mencionados autores afirmam que mais 

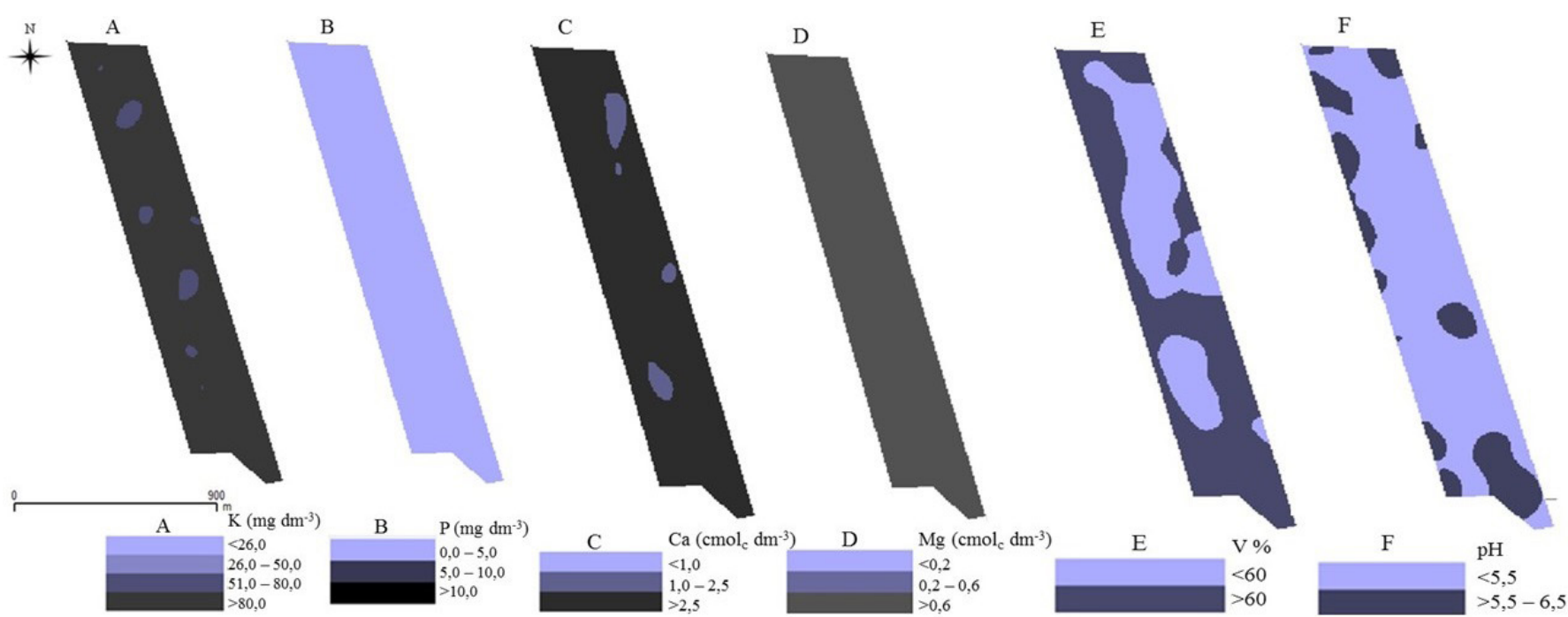

Figura 2. Mapas de krigagem dos atributos químicos potássio (A), fósforo (B), cálcio (C), magnésio (D), saturação por bases (E) e pH (F) de um Latossolo Vermelho amarelo em área de cultivo de algodoeiro em sistema plantio direto

pesquisas são necessárias para determinar se a economia de fertilizantes é suficiente para compensar os custos adicionais de amostragem e análise do solo e equipamentos especializados que a aplicação taxa variável de fertilizantes exige.

Ressalta-se a importância da geração de mapas de krigagem com as classes dos atributos baseadas em recomendações de fertilidade do solo visto que no caso do fósforo, mesmo apresentando forte dependência espacial, na geração do mapa por krigagem, $100 \%$ da área estavam inseridos na classe de teores classificados "muito baixo", ou seja, homogêneo em toda a área, segundo classificação de Carvalho et al. (2011).

Com relação ao teor de $\mathrm{K}$ observa-se, na Figura 2A, que $4,1 \% \mathrm{da}$ área possuem teor de $\mathrm{K}$ entre 51 e $80 \mathrm{mg} \mathrm{dm}^{-3} \mathrm{e} 95,9 \%$ da área estão com teor de $\mathrm{K}>80 \mathrm{mg} \mathrm{dm}^{-3}$, demonstrando maior desuniformidade se comparado ao $P$ porém ainda insignificante para uso da agricultura de precisão e aplicação em taxa variável. Para a cultura do algodoeiro no cerrado, os teores de K neste solo são considerados adequado/bom em $4,1 \%$ da área e alto/muito bom em 95,9\% da área (Carvalho et al., 2011). Desta forma, a adubação de manutenção com o K, em conjunto com a dose de reposição em função da expectativa de produtividade da área, seria suficiente para manter os níveis de $\mathrm{K}$ adequados no solo não sendo vantajoso fazer a aplicação em taxa variável uma vez que a variação dos teores no solo foi muito baixa.

Para o teor de cálcio $(\mathrm{Ca})$ no solo (Figura $2 \mathrm{C}$ ) observou-se que $4,9 \%$ da área possuem teor de Ca de 1 a $2,5 \mathrm{cmol}_{c} \mathrm{dm}^{-3}$ (classificado como médio) e 95,1\% da área apresentam teor de cálcio maior que 2,5 $\mathrm{cmol}_{c} \mathrm{dm}^{-3}$ (classificado como alto) (CFSG, 1988). O Magnésio (Figura 2D) apresentou níveis superiores a $0,6 \mathrm{cmol}_{\mathrm{c}} \mathrm{dm}^{-3} \mathrm{em} 100 \%$ da área os quais são classificados, no solo, como "alto" em toda a área; a exemplo do que foi verificado com os nutrientes fósforo e potássio, a aplicação em taxa variável para cálcio e magnésio também seria desnecessária.

Quanto ao pH (Figura 2F) apresentou maior heterogeneidade sendo $78,9 \%$ da área com $\mathrm{pH}<5,5$ e 21,1\% com pH > 5,5. Os valores da saturação por bases (V\%) (Figura 2E), foram classificados em 38,4\% da área com V\% < 60 e 61,6\% da área com
V\% > 60. Segundo Carvalho et al. (2011) existem evidências de que a cultura do algodoeiro responde positivamente à calagem no Cerrado até a saturação por bases de $60 \%$ verificando-se, então, que nesta área é notória a necessidade de se realizar a calagem, em parte da área, a fim de se elevar a saturação de bases até $60 \%$. É sumamente importante ressaltar que a alteração na saturação por bases no solo é lenta podendo não ser alcançada a meta no mesmo ano de aplicação do calcário devido à acidificação natural do solo pela mineralização da matéria orgânica, adição de fertilizantes nitrogenados, perdas por lixiviação e absorção e acidificação do solo pelas raízes das plantas. Desta forma, poderia ser aplicado calcário em toda a área, em doses diferentes em função das áreas de manejo determinadas pelo mapa gerado.

Analisando a heterogeneidade nos valores de $\mathrm{pH}$ e V\%, infere-se que nesta área seria viável a introdução da aplicação de calcário em taxa variável visto que bons resultados poderiam ser obtidos em economia de calcário e melhor uniformidade na fertilidade do solo. Este resultado está de acordo com as afirmações de Souza et al. (2007) que comprovaram a eficiência da correção da acidez do solo por meio da aplicação de calcário em taxa variável utilizando da análise geoestatística como técnica para determinação da heterogeneidade do solo.

Com a elevada acidez $(\mathrm{pH}<5,5)$ e saturação de bases (V\%) inferior a 60 em 78,9 e 38,4\% da área, respectivamente, fica evidenciada a necessidade de realização de calagem na área objetivando a correção da acidez e a homogeneização da fertilidade do solo de maneira que as culturas agrícolas possam responder satisfatoriamente em produtividade. Segundo Carvalho et al. (2011) a acidez dos solos afeta negativamente o desenvolvimento radicular e o crescimento do algodoeiro refletindo diretamente na produtividade, sendo encontrados relatos de queda na produtividade do algodoeiro em solos com pH inferior a 5,5. Em estudo desenvolvido por Corá et al. (2004) a avaliação da variabilidade espacial de atributos químicos do solo na cultura de cana-de-açúcar possibilitou definir zonas homogêneas de manejo específico com o objetivo 
de efetuar a aplicação de calcário e fertilizantes a taxas variáveis proporcionando, desta forma, um manejo mais eficiente e econômico da cultura.

De maneira geral, os resultados indicaram variabilidade espacial dos atributos químicos do solo com teores variando desde a classe baixa até a classe alta para alguns atributos. Por outro lado, os teores de fósforo, de suma importância para a produção agrícola em solos do Cerrado, se apresentaram homogêneos em toda a área quando colocados os valores em classes de fertilidade do solo, classificado como "muito baixo", indicando a necessidade da adubação fosfatada na área para obtenção de elevadas produtividades.

A recomendação da utilização de técnicas de agricultura de precisão e a aplicação de insumos em taxa variável devem ser realizadas de maneira criteriosa visto que, na área em estudo e apesar de alguns atributos, como o $\mathrm{P}$ e o $\mathrm{K}$, $\mathrm{Ca}$ e $\mathrm{Mg}$ apresentarem dependência espacial e variabilidade dentro da área quando elaborados mapas categorizados em classes de fertilidade do solo, os mesmos não indicam a necessidade de aplicação em taxa variável para esses nutrientes; por outro lado, atributos como o pH e V\% apresentaram, após gerados os mapas de classes de fertilidade do solo, potencialidade para a realização de calagem em aplicação com taxa variável demonstrando que dentro de uma mesma área podem existir diversas situações distintas sendo recomendada ou não, a adoção do manejo seguindo os preceitos da agricultura de precisão.

\section{Conclusões}

1. A dependência espacial encontrada nas propriedades químicas do solo analisadas indica que as variações espaciais devem ser consideradas no planejamento de coleta de amostras de solo e nas práticas de manejo do solo.

2. Dentre os atributos analisados o teor de fósforo foi o que apresentou maior coeficiente de variação.

3. Para a área em estudo a aplicação de insumos em taxa variada seria recomendada apenas para a aplicação de calcário devido à heterogeneidade apresentada nos mapas de V\% e pH.

\section{Literatura Citada}

Aimrun, W.; Amim, M. S. M.; Ahmad, D.; Hanafi, M. M.; Chan, C.S. Spatial variability of bulk soil electrical conductivity in a Malaysian paddy field: key to soil management. Paddy Water Environmental, v.5, p.113-121, 2007.

Akaike, $\mathrm{H}$. Information theory as extension of the maximum likelihood principle. In: International Simposium on Information Theory, 2, 1973, Budapest. Anais... Budapest: Akadêmia Kiadó, 1973. p.267-281.

Amado, T. J. C.; Pes, L. Z.; Lemainski, C. L.; Schenato, R. B. Atributos químicos e físicos de Latossolos e sua relação com os rendimentos de milho e feijão irrigados. Revista Brasileira de Ciência do Solo, v.33, p.831-843, 2009.

Andrade, A. R. S.; Guerrini, I. A.; Garcia, C. J. B.; Katez, I.; Guerra, H. O. C. Variabilidade espacial da densidade do solo sob manejo da irrigação. Ciência e Agrotecnologia, v.29, p.322-329, 2005.
Bronson, K.; Keeling, W.; Booker, J. D.; Chua, T.; Wheeler, T.; Boman, R.; Lascano, R. Influence of landscape position, soil series, and phosphorus fertilizer on cotton lint yield. Agronomy Journal, v.95, p.949-957, 2003.

Cambardella, C. A.; Moorman, T. B.; Nowak, J. M.; Parkin, T. B.; Karlen, D. L.; Turco, R. F.; Konopka, A. E. Field-scale variability of soil properties in central Iowa soils. Soil Science Society American Journal, v.58, p.1501-1511, 1994.

Carvalho, M. C. S.; Ferreira, G. B.; Staut, L. A. Nutrição, calagem e adubação do algodoeiro. In: Freire, E. C. (Editor). Algodão no cerrado do Brasil. 2.ed. Aparecida de Goiânia: Mundial Gráfica, 2011, 1082p.

Carvalho, M. P.; Takeda, E. Y.; Freddi, O. S. Variabilidade espacial de atributos de um solo sob videira em Vitória Brasil (SP). Revista Brasileira de Ciência do Solo, v.27, p.695-703, 2003.

CFSG - Comissão de Fertilidade de Solos de Goiás. Recomendações de corretivos e fertilizantes para Goiás. 5a Aproximação. Goiânia: UFG/EMGOPA, 1988, 101p. Convênio. Informativo Técnico, 1

Chaves, L. H. G.; Farias, C. H. A. Variabilidade espacial de cobre e manganês em Argissolo sob cultivo de cana-de-açúcar. Revista Ciência Agronômica, v.40, p.211-218, 2009.

Corá, J. E.; Araújo, A. V.; Pereira, G. T.; Beraldo, J. M. G. Variabilidade espacial de atributos do solo para adoção do sistema de agricultura de precisão na cultura de cana-deaçúcar. Revista Brasileira de Ciência do Solo, v.28, p.10131021, 2004.

Corá, J. E.; Beraldo, J. M. G. Variabilidade espacial de atributos do solo antes e após calagem e fosfatagem em doses variadas na cultura de cana-de-açúcar. Engenharia Agricola, v.26, p.374-387, 2006.

Dalchiavon, F. C.; Carvalho, M. de P. e; Andreotti, M.; Montanari, R. Variabilidade espacial de atributos da fertilidade de um Latossolo Vermelho Distroférrico sob Sistema Plantio Direto. Revista Ciência Agronômica, v.43, p. 453-461, 2012.

Dalchiavon, F. C.; Carvalho, M. P. e; Nogueira, D. C.; Romano, D.; Abrantes, F. L.; Assis, J. T.; Oliveira, M. S. Produtividade da soja e resistência mecânica à penetração do solo sob sistema plantio direto no cerrado brasileiro. Pesquisa Agropecuária Tropical, v.41, p.8-19, 2011.

Faria, G. E.; Barros, N. F.; Novais, R. F.; Silva, I. R. Soil fertility, organic carbon and fractions of the organic matter at different distances from eucalyptus stumps. Revista Brasileira de Ciência do Solo, v.33, p.571-579, 2009.

Gomes, F. P.; Garcia, C. H. Estatística aplicada a experimentos agronômicos e florestais: Exposição com exemplos e orientações para uso de aplicativos. Piracicaba: FEALQ, 2002. 309p.

Inamassu, R. Y.; Bernardi, A. C. de C.; Vaz, C. M. P.; Naime, J. de M.; Queiros, L. R.; Resende, A. V. de; Vilela, M. de F.; Jorge, L. A. de C.; Bassoi, L. H.; Perez, N. B.; Fragalle, E. P. Agricultura de Precisão para a sustentabilidade de sistemas produtivos do agronegócio brasileiro. In: Inamassu, R. Y.; Naime, J. de M.; Resende, A. V. de; Bassoi, L. H.; Bernardi, A. C. de C. (ed.). Agricultura de precisão: Um novo olhar. São Carlos: Embrapa Instrumentação, 2011. 344p. 
Inamassu, R. Y.; Simões, M. S.; Luchiari júnior, A.; Rabello, L. M.; Molin, J. P. Sistema de medida de condutividade elétrica do solo adaptado a um implement agrícola (subsolador). São Carlos: Embrapa Instrumentação Agropecuária, 2007. 5p. Circular Técnica, n.40

Lemos Filho, L. C. A.; Oliveira, E. L. de; Faria, M. A. de; Andrade, L. A. de B. Variação espacial da densidade do solo e matéria orgânica em área cultivada com cana-de-açúcar (Saccharum officinarum L.). Revista Ciência Agronômica, v.39, p.193-202, 2008.

Minasny, B.; Mcbratney, A. B.; Whelan, B. M. 2002. Vesper version 1.6. Sidney: Australian Centre for Precision Agriculture, McMillan Building A05, The University of Sydney, NSW, 2006.

Motomiya, A. V. A.; Molin, J. P.; Motomiya, W. R.; Vieira, S. R. Spatial variability of soil properties and cotton yield in the Brazilian Cerrado. Revista Brasileira de Engenharia Agrícola e Ambiental, v.15, p.996-1003, 2011.

Quantum Gis Development Team. Quantum GIS Geographic Information System. Open Source Geospatial Foundation Project. <http://qgis.osgeo.org> 3 Jul. 2012.

Serrano, J. M.; Peça, J. O.; Silva, J. R.; Shahidian, S. Medição e mapeamento da condutividade elétrica aparente do solo em pastagens. Revista Ciências Agrárias, v.33, p.5-14, 2010.

Shapiro, S. S.; Wilk, M. B. An analysis of variance test for normality (complete samples). Biometrika, v.52, p.591-611, 1965.
Silva, P. C. M.; Chaves, L. H. G. Avaliação e variabilidade espacial de fósforo, potássio e matéria orgânica em Alissolos. Revista Brasileira de Engenharia Agrícola e Ambiental, v.5, p.431436, 2001

Souza, Z. M.; Barbieri, D. M.; Marques Júnior, J.; Pereira, G. T.; Campos, M. C. C. Influência da variabilidade espacial de atributos químicos de um latossolo na aplicação de insumos para cultura de cana-de-acúcar. Ciência e Agrotecnologia, v.31, p.371-377, 2007.

Stewart, C.; Boydell, B.; McBratney, A. Precision decisions for quality cotton: A guide to site-specific cotton crop management. Sidney, University of Sydney, 2005. 107p.

Veris Technologies. <http://www. veristech.com>. 2 Set. 2011.

Vieira, V. A. S.; Mello, C. R.; Lima, J. M. Variabilidade espacial de atributos físicos do solo em uma microbacia hidrográfica. Ciência e Agrotecnologia, v.31, p.1477-1485, 2007.

Werner, V;; Schlosser, J. F.; Rozin, D.; Pinhero, E. D.; Dornelles, M. E. C. Aplicação de fertilizantes a taxa variável em agricultura de precisão variando a velocidade de deslocamento. Revista Brasileira de Engenharia Agrícola e Ambiental, v.11, p.658663, 2007.

Zanão Júnior, L. A.; Lana, R. M. Q.; Carvalho-Zanão, M. P.; Guimarães, E. C. Variabilidade espacial de atributos químicos em diferentes profundidades em um Latossolo em sistema de plantio direto. Revista Ceres, v.57, p.429$438,2010$. 